\title{
Estimation of fruit and vegetable consumption in a cohort of Ghanaian women and evaluation of knowledge, attitudes and practice
}

\author{
J. Moss ${ }^{1,2}$ and S. Mushtaq ${ }^{2}$ \\ ${ }^{1}$ Healthbox Community Interest Company, Cheshire, CH2 4HX and ${ }^{2}$ Department of Clinical Sciences and Nutrition, \\ University of Chester, Chester, CHI 4BJ, UK
}

Non-communicable diseases (NCDs), previously confined to industrialised nations, are spreading through the developing world at unprecedented rates ${ }^{(1)}$. With communicable diseases still prevalent, this imposes a double-burden of disease in countries with limited resources and ill-equipped health systems ${ }^{(2)}$. An unhealthy diet, including insufficient consumption of fruit and vegetables is one of four main behavioural risk factors in the development of NCDs. However, consumption across the world, including Ghana, is below recommended levels ${ }^{(3)}$. Despite a wealth of research in developed countries, few studies have investigated barriers to fruit and vegetable consumption in Ghana. The aim of the present study was to assess fruit and vegetable consumption and evaluate knowledge, attitudes and practice in a cohort of Ghanaian women.

A mixed-methods approach, incorporating a survey delivered in a guided interview format was utilised to investigate patterns and determinants of fruit and vegetable consumption in a cohort of Ghanaian women. Data were collected from female environmental health and nursing students aged 18-33 years $(n=74$, response rate 98.7\%), residing in the Korle-Bu district of Accra, Ghana. A 24-hour recall questionnaire, local handy measures and a specially designed portion size assessment sheet allowed estimation of fruit and vegetable consumption, whilst a questionnaire comprising both qualitative and quantitative questions enabled investigation of barriers to consumption in Ghanaian society.

A significantly greater level of low fruit and vegetable consumption was found in the present study (69\%), than detailed in the World Health Survey 2002-03 $(p \leq 0.001)$. There was a significant positive association between meal frequency and consumption levels $(p=0.025)$, however, no association was found between consumption and knowledge levels, income, home-production or perceived adequate consumption. Themes emerging from the research related to barriers affecting consumption included cost, availability, quality, and health and safety issues. Unhealthy snacking was found to be common, as were unhealthy substitutions during periods of scarcity.

Numerous barriers causing the observed decrease in fruit and vegetable consumption were identified in the present study. The interrelating nature of the barriers identified suggests a multidirectional approach to address these issues would offer the greatest benefits for consumption levels. Increasing agricultural infrastructure is key, whilst educational initiatives should also play a major role in future strategies to increase fruit and vegetable consumption. Future investment and policy in Ghana should focus on prevention rather than cure, if the growing NCD burden is to be halted.

This work was partially funded by the Denis Burkitt Study Award 2010.

1. Popkin BM, Adair LS \& Ng SW (2012) Nutr Rev 70, 3-21.

2. Boutayeb A \& Boutayeb S (2005) Int J Equity Health 4, 2.

3. Hall JN, Moore S, Harper, SB \& Lynch JW (2009) Am J Prev Med 36, 402-409. 